\title{
Scaling supernova hydrodynamics to the laboratory*
}

J. Kane ${ }^{\dagger, a)}$ and D. Arnett

University of Arizona, Tucson, Arizona 85721

B. A. Remington, S. G. Glendinning, and G. Bazan

Lawrence Livermore National Laboratory, Livermore, California 94550

R. P. Drake

University of Michigan, Ann Arbor, Michigan 48109-2143

B. A. Fryxell

ASCI Flash Center, University of Chicago, Chicago, Illinois 60637

R. Teyssier

CEA/DSM/DAPNIA/Service d'Astrophysique, Bat. 709 Centre d'Etudes de Saclay L'Orme des Merisiers F-91191 Gif-sur-Yvette Cedex, France

K. Moore

Harvey Mudd College, Claremont, California 91711

(Received 19 November 1998; accepted 18 January 1999)

Supernova (SN) 1987A focused attention on the critical role of hydrodynamic instabilities in the evolution of supernovae. To test the modeling of these instabilities, we are developing laboratory experiments of hydrodynamic mixing under conditions relevant to supernovae. Initial results were reported in J. Kane et al. [Astrophys. J. 478, L75 (1997) and B. A. Remington et al., Phys. Plasmas 4, 1994 (1997)]. The Nova laser is used to generate a 10-15 Mbar shock at the interface of a two-layer planar target, which triggers perturbation growth due to the Richtmyer-Meshkov instability, and to the Rayleigh-Taylor instability as the interface decelerates. This resembles the hydrodynamics of the He-H interface of a Type II supernova at intermediate times, up to a few $\times 10^{3} \mathrm{~s}$. The scaling of hydrodynamics on microscopic laser scales to the $\mathrm{SN}$-size scales is presented. The experiment is modeled using the hydrodynamics codes HYADES [J. T. Larson and S. M. Lane, J. Quant. Spect. Rad. Trans. 51, 179 (1994)] and CALE [R. T. Barton, Numerical Astrophysics (Jones and Bartlett, Boston, 1985), pp. 482-497], and the supernova code PROMETHEus [P. R. Woodward and P. Collela, J. Comp. Phys. 54, 115 (1984)]. Results of the experiments and simulations are presented. Analysis of the spike-and-bubble velocities using potential flow theory and Ott thin-shell theory is presented, as well as a study of 2D versus 3D differences in perturbation growth at the He-H interface of SN 1987A. (C) 1999 American Institute of Physics.[S1070-664X(99)93205-6]

\section{INTRODUCTION}

Observations of SN 1987A, a core collapse supernova $(\mathrm{SN})$ in the Large Magellanic Cloud, strongly suggested the occurrence of material mixing driven by the RichtmyerMeshkov (RM) and Rayleigh-Taylor (RT) instabilities. ${ }^{1-5}$

The "Bochum event," $6-8$ and early detection of radioactive ${ }^{56} \mathrm{Co}$ from the explosively burned oxygen layer, implied that the ${ }^{56} \mathrm{Co}$ had been mixed well into the outer layers. Doppler broadening of the gamma-ray, optical, and infrared lines from ${ }^{56} \mathrm{Co},{ }^{56} \mathrm{Ni}$, and ${ }^{56} \mathrm{Fe}$ implied velocities in excess of $3000 \mathrm{~km} / \mathrm{s},{ }^{9-11}$ whereas two-dimensional (2D) modeling to date predicts maximum velocities of $\leqslant 2000 \mathrm{~km} / \mathrm{s}$, suggesting that perhaps 3D hydrodynamics effects should be considered. Given the fundamental role played by the RM

*Paper F3I1.3 Bull. Am. Phys. Soc. 43, 1702 (1998).

'Invited speaker.

${ }^{a)}$ Present address: Lawrence Livermore National Laboratory, Livermore, California 94550. and RT instabilities in SN evolution, it is desirable to develop the means of testing the hydrodynamics of the $\mathrm{SN}$ codes. We report here on experiments using the Nova laser at Lawrence Livermore National Laboratory (LLNL) to test the modeling of compressible RM and RT instabilities at relevant pressures and under scaled conditions. We use the SN code PROMETHEUS to model the experiment, and for comparison, the LLNL code CALE. Initial results were presented in Refs. 12 and 13. For discussion of other astrophysically relevant sets of experiments at the Nova laser, see Refs. 13, 14 , and 15. In the present paper we also discuss scaling of the hydrodynamics from supernovae to the laboratory, present an analysis of the hydrodynamic growth in the experiment in terms of theory for incompressible hydrodynamic instabilities, and report on numerical investigations of $2 \mathrm{D}$ versus $3 \mathrm{D}$ differences in the hydrodynamic instability growth in SN 1987A.

We model the laser experiments and SN 1987A using a combination of codes: HYADES, CALE, and PROMETHEUS. The 

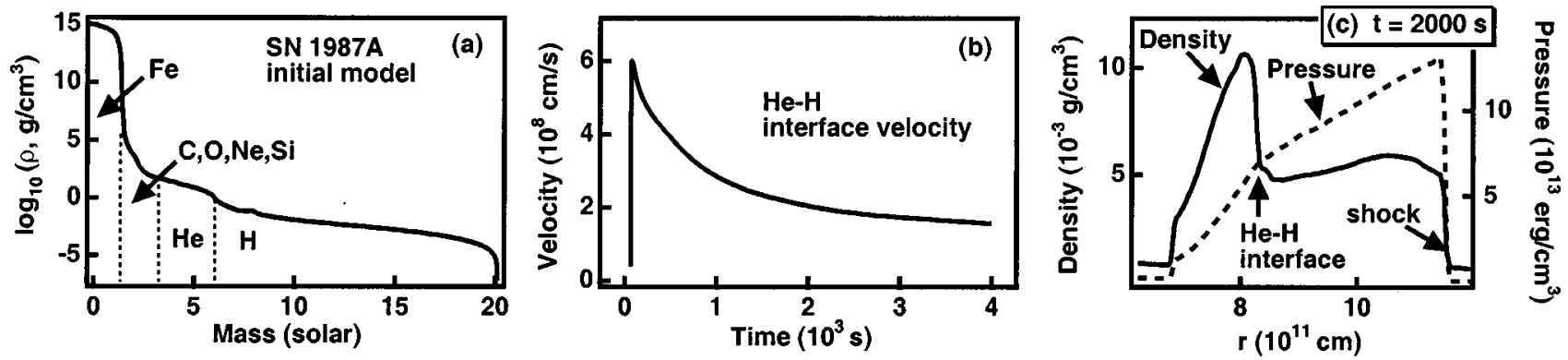

FIG. 1. (a) Initial model for SN 1987A; (b) He-H interface velocity; (c) crossed density and pressure gradients at the He-H interface after passage of the shock.

HYADES code (see Ref. 16) is a 1D Lagrangian code with multigroup radiation transport and tabular Equation of State (EOS); CALE is a 2D Arbitrary Lagrangian Eulerian (ALE) code (see Ref. 17) with tabular EOS and interface tracking; CALE allows both planar and cylindrical $(r-z)$ geometry. PROMETHEUS is a multi-D hydrodynamics code which uses the Piecewise Parabolic Method (PPM; see Ref. 18). PROMETHEUS allows planar, spherical, and cylindrical geometry, and can be run in 1D, 2D, or 3D. Ideal gas EOS is used for all the PROMETHEUS simulations of the laser experiments; we choose an adiabatic exponent $\gamma$ for each material in the target to best reproduce the 1D shock speed, and velocity of the interface between the materials, as simulated by HYADES and CALE using tabular EOS. We use HYADES to model the input of laser energy into the target, before mapping to CALE and PROMETHEUS and continuing the simulations in two or three dimensions (2D or $3 \mathrm{D}$ ). The versions of CALE and PROMETHEUS that we are using do not have radiation transport. We use mainly CALE to design the laser experiments, because we can use tabular EOS with CALE. We use PROMETHEUS to do low resolution simulations of the laser targets which have $3 \mathrm{D}$ features and require a $3 \mathrm{D}$ code. We use PROMETHEUS in spherical geometry to simulate SN 1987A.

\section{1D SIMULATIONS OF SN AND LASER EXPERIMENT}

Figure 1(a) shows a 20 solar mass model for the progenitor of SN 1987A (see Ref. 19). Using the PROMETHEUS code, which was described in Sec. I, we show in Fig. 1(b) the 1D velocity profile of the $\mathrm{He}-\mathrm{H}$ interface during the intermediate stages of the explosion, and in Fig. 1(c) the 1D pressure and density profiles $2000 \mathrm{~s}$ into the explosion. We consider these intermediate stages of the explosion, which follow the early stages in which the core collapses and rebounds, to end at about $12 \times 10^{3}$ to $15 \times 10^{3} \mathrm{~s}$, by which time the blast wave has left the star and a rarefaction has moved back inward from the surface into the outer layers. For more details of the SN 1987A explosion, the PROMETHEUS code, and the simulations shown here, see Refs. 5, 12, 13, 18 and 20-22. At the $\mathrm{He}-\mathrm{H}$ interface, the strong acceleration induced by the blast wave, followed by the deceleration caused by the crossed pressure and density gradients, suggest that the $\mathrm{He}-\mathrm{H}$ interface should exhibit strong RM and RT instability. Simulations in 2D with PROMETHEUS show instabilities at the $\mathrm{He}-\mathrm{H}$ and $\mathrm{He}-\mathrm{O}$ interfaces evolving well into the nonlinear regime; see Refs. 20-22.

The Nova experimental configuration is illustrated in Fig. 2(a); for further discussion of the Nova laser, the experiment, and the experimental techniques, see Refs. 12, 13 and 23-26 and references therein. The Nova laser is used to produce an $\mathrm{X}$-ray drive which shocks a two-layer $\mathrm{Cu}-\mathrm{CH}_{2}$ planar target having an imposed material perturbation at the $\mathrm{Cu}-\mathrm{CH}_{2}$ interface. Figures 2(b) and 2(c) show HYADES simulations of a 1D (unperturbed) experiment; as in the SN case (Fig. 1), the interface is first accelerated by the shock and then decelerated, because the pressure and density gradients are crossed at the interface; the $\mathrm{Cu}-\mathrm{CH}_{2}$ should exhibit strong $\mathrm{RM}$ and RT instability, the same as the He-H interface in SN 1987A (compare Figs. 1 and 2). We typically observe the experiment for up to $40 \mathrm{~ns}$.

The numerical codes we use were described in Sec. I. We begin all simulations using HYADES, then map to PROMETHEUS and CALE at $2.45 \mathrm{~ns}$, just prior to the arrival of the shock at the thinnest part of the (perturbed) $\mathrm{Cu}$. We use
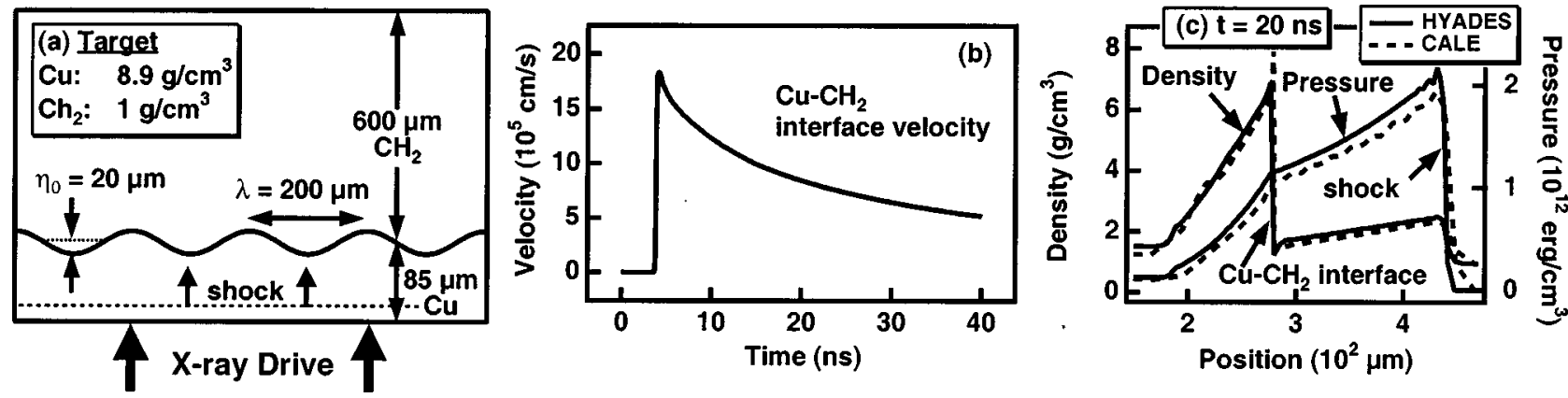

FIG. 2. (a) Initial target for Nova experiment; (b) $\mathrm{Cu}_{-} \mathrm{CH}_{2}$ interface velocity; (c) crossed density and pressure gradients at the $\mathrm{Cu}-\mathrm{CH}_{2}$ interface. 


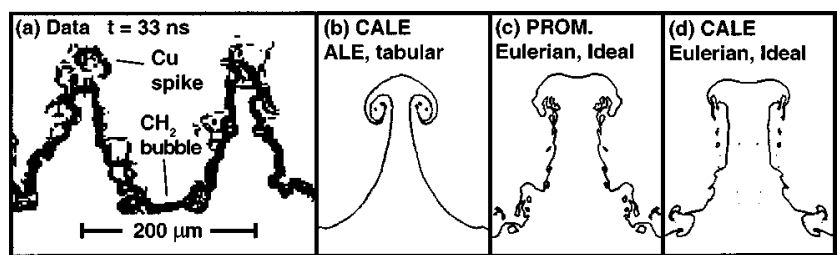

FIG. 3. Data and simulations for $2 \mathrm{D}$ experiment. (a) Outline of $\mathrm{Cu}-\mathrm{CH}_{2}$ interface extracted from radiograph of experiment at $33 \mathrm{~ns}$; (b) CALE simulation, in (ALE) mode and using tabular EOS; (c) PROMETHEUs simulation, with fixed (Eulerian) orthogonal grid and ideal gas EOS. There is more fine structure with PROMETHEUS; (d) when CALE is run with Eulerian orthogonal grid and ideal gas EOS, the result is similar to PROMETHEUS.

the measured x-ray radiation temperature, $T_{r}(t)$, as the energy input to HYADES, and the versions of CALE and PROMETHEUS that we are using do not have radiation transport. As seen in Fig. 2(c), CALE and HYADES agree well long after the mapping, indicating that the experiment is hydrodynamics.

\section{RESULTS AND 2D SIMULATIONS}

Figure 3(a) shows a 2D image from the experiment at $t=33.2 \mathrm{~ns}$. The $\mathrm{Cu}-\mathrm{CH}_{2}$ interface shows the classic nonlinear RM/RT bubble-and-spike shape, and there are faint indications of a roll-up at the very tip of the spike. We initiate our 2D simulations in the same manner as in 1D: we map the conditions from the HYADES calculation at $2.45 \mathrm{~ns}$. Figures 3(a)-3(d) show PROMETHEUS and CALE simulations of the experiment. In PROMETHEUS, ideal gas EOS was used, with $\gamma_{\mathrm{Cu}}=46 / 30$ and $\gamma_{\mathrm{CH}_{2}}=65130$. In the Eulerian PROMETHEUS and CALE simulations, the zones are $1 \mu \mathrm{m}$ square, which gives a resolution of 200 zones per wavelength of the perturbation. In the CALE ALE mode simulation, not all initial zones are square, because the initial interface is represented as a smooth perturbation. There are 200 zones per wavelength parallel to the interface. In the direction perpendicular to the interface, the depth of a zone is 1 $\mu \mathrm{m}$ near the interface, and zones are feathered outward to larger size in that direction toward the far end of the $\mathrm{CH}_{2}$ (away from the interface); an effective resolution of $1 \mu \mathrm{m}$ square per zone is maintained in the region of interest (where the bubbles and spikes form) because of the natural compression of zones in ALE mode. The gross features of the experiment are well reproduced by both simulations. For further details about the data and simulations, see Refs. 12 and 13 . The differences in fine structure and shape of the spike tip vortex among the simulations is primarily due to stairstepping the initial interface, as opposed to representing it as a smooth sinusoid. Figure 4 compares spike-and-bubble position from the experiment, CALE, and PROMETHEUS. The observed spike-and-bubble fronts are well reproduced by both hydrodynamics codes. Also shown is the position of an unperturbed interface as calculated by CALE and PROMETHEUS. The bubble-and-spike velocities we discuss in the following are with respect to this unperturbed interface. In Ref. 12 we presented a preliminary analysis of the hydrodynamic growth in terms of nonlinear RM and RT theory for semi-infinite fluids (see Refs. 27-29). In more detailed analyses we next

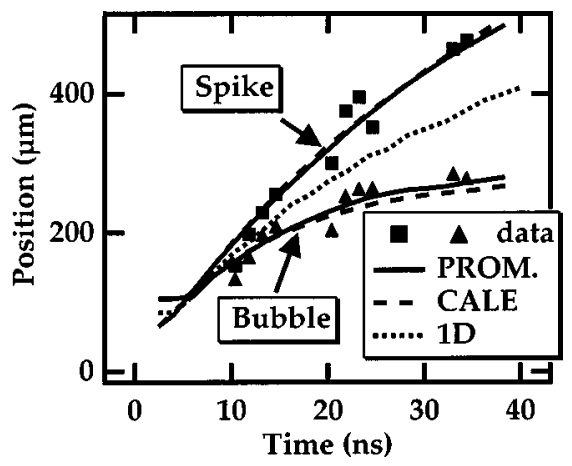

FIG. 4. Bubble-and-spike velocities.

consider the effects of the "accordion-like" decompression of the $\mathrm{Cu}$ and $\mathrm{CH}_{2}$ layers, the effects of the time varying effective $g$ (interface deceleration), and the finite thickness of the dense part of the Cu layer [see Fig. 2(c)].

In the following we compare predictions for bubble-andspike velocities from potential flow theory and our modification of the Ott thin-shell theory (see Refs. 30 and 31 for the thin-shell theory) to the results of a CALE simulation of the Nova experiment. We use Meyer-Blewet (MB) theory (see Refs. 24 and 32) for the linear stage of bubble-and-spike growth, to provide initial conditions for the potential flow and thin-shell theories. The justification for considering thinshell theory is seen in Fig. 2(c); in terms of density, the half-height thickness of the dense $\mathrm{Cu}$ is small at $20 \mathrm{~ns}-$ about $50 \mu \mathrm{m}-$ compared to the perturbation wavelength $\lambda$ $=200 \mu \mathrm{m}$. By comparison, the shocked $\mathrm{CH}_{2}$ layer is comparable in thickness to $\lambda$. The original Ott theory is for a thin-shell surrounded above and below by massless fluids at constant pressure. In our application of the theory, the fluids above and below are not massless; below the thin dense $\mathrm{Cu}$ shell there is low density $\mathrm{Cu}$, and above the dense $\mathrm{Cu}$ shell there is low density $\mathrm{CH}_{2}$. To account for this, we do an $\mathrm{ad}$ hoc modification of the thin-shell theory, inserting the postshock Atwood number $A^{*} \equiv\left(\rho_{\text {heavy }}^{*}-\rho_{\text {light }}^{*}\right) /\left(\rho_{\text {heavy }}^{*}+\rho_{\text {light }}^{*}\right)$ into the solution of the thin-shell equations, where the* indicates post-shock values.

We "undecompress" the CALE bubble-and-spike velocities before doing the comparisons, to account for the decompression of the fluids over time. We do so by subtracting $u_{1}(x, t)$, the fluid velocity with respect to the interface in a 1D (unperturbed) simulation, from each bubble or spike velocity $u_{x}(x, y, t)$, where $x$ and $y$ are the coordinate directions perpendicular and parallel to the 1D interface, respectively. For the modified thin-shell theory, we use the predictions of MB to determine the amplitude $\eta_{0}$ and velocity $\partial \eta / \partial t_{0}$ of the perturbation just after the shock has passed the perturbed 2D interface. The Ott theory is correct for the thin shell throughout the linear and nonlinear stages, and we apply it as early as possible, to account for the effect of $A^{*}$ in our modified theory. For the potential flow theory, we take as initial conditions the prediction of $\mathrm{MB}$ for $\partial \eta / \partial t$ at a time $t_{1}$ when the initial perturbation has inverted (because of the RM instability) and $\eta\left(t_{1}\right) / \lambda=0.1$, so that the growth is becoming nonlinear. We map from $\mathrm{MB}$ at the later time $t_{1}$ because the 
drag-vs-buoyancy theory assumes that the perturbation has some "shape," represented by drag and buoyancy coefficients. In the laser experiment, the effective deceleration $g(t)$ of the interface is fit extremely well by the simple form $g(t)=\alpha / t$, where $t=0$ is the time at which the shock hits the 1D (unperturbed interface); for the correct $\alpha$, we integrate $g(t)$ over time to reproduce the interface velocity and position. A value of $\alpha \approx 3.1 \mu \mathrm{m} / \mathrm{ns}$ reproduces these trajectories extremely well.

Following Refs. 30 and 31, the thin-shell equations are

$$
\ddot{x}(\xi, t)=-g(t) z^{\prime}(\xi, t), \quad \ddot{z}(\xi, t)=-g(t) x^{\prime}(\xi, t),
$$

where $\xi$ is the Lagrangian coordinate of a point on the thin shell, and $x$ and $z$ are the Eulerian coordinates parallel and perpendicular, respectively, to the unperturbed interface. In his original paper (Ref. 30), Ott presented the solution for constant $g$. In Ref. 31, Basko presented the solution for the case $g(t) \propto 1 / t^{2}$. We present here the solution for the case $g(t)=\alpha / t$, namely

$$
\begin{aligned}
& x(\xi, t)=\xi+f(t) \cos (k \xi), \quad z(\xi, t)=z_{0}+f(t) \sin (k \xi), \\
& \ddot{z}_{0}(t)=g(t), \\
& f(t)=\sqrt{\frac{t}{A^{*} k \alpha}}\left\{a_{1} I_{1} \sqrt{A^{*} k \alpha t}-b_{1} K_{1} \sqrt{A^{*} k \alpha t}\right\},
\end{aligned}
$$

where $k=2 \pi / \lambda$ is the wave number of the perturbation, and $I_{1}$ and $K_{1}$ are the growing and damped modified Bessel functions, respectively. The two constants $a_{1}$ and $b_{1}$ are set by the amplitude and velocity of the initial perturbation. In the exact solution, with massless fluids above and below, the post-shock Atwood number $A^{*}$ is one. To crudely account for the arbitrary post-shock Atwood number, we insert $A^{*}$ back into the $A=1$ solution, replacing all factors of $k \alpha$ with $A^{*} k \alpha$.

Following Refs. 28 and 29, the drag-vs-buoyancy potential flow equation for the velocities of the bubble and spike is

$$
\dot{u}(t)=c_{b} g(t)-c_{D} u(t)^{2} / \lambda,
$$

where the buoyancy coefficient is $c_{b}=A^{*} /\left(1+A^{*}\right)$ and the drag coefficient is $c_{D \text {,buble }}=3 \pi$ for the bubble and $c_{D \text {, spike }}$ $=3 \pi\left(1-A^{*}\right) /\left(1+A^{*}\right)$ for the spike. We use MB, as described above, to determine the initial value $u\left(t_{1}\right)$ and then integrate Eq. (1) over time.

The result of mapping MB to potential flow and to the modified Ott theory is shown in Fig. 5. Figure 5(a) shows the undecompressed bubble-and-spike velocities versus time from a 2D CALE simulation of the experiment, the result of MB mapped to potential flow theory for the spike and bubble, and the result of $\mathrm{MB}$ mapped to the modified thinshell theory for the bubble. MB mapped to potential flow theory predicts the spike velocity reasonably well. The modified thin-shell theory predicts the bubble velocity well at early times, while at later times the potential flow theory predicts the bubble velocity better. In Fig. 5(b) we show the prediction of MB mapped to potential flow theory for the spike velocity minus bubble velocity; we show the results of integrating Eq. (1) both with $g(t) \propto \alpha / t$ and with $g(t)$ set to zero. In the former case we account for both the RM insta-
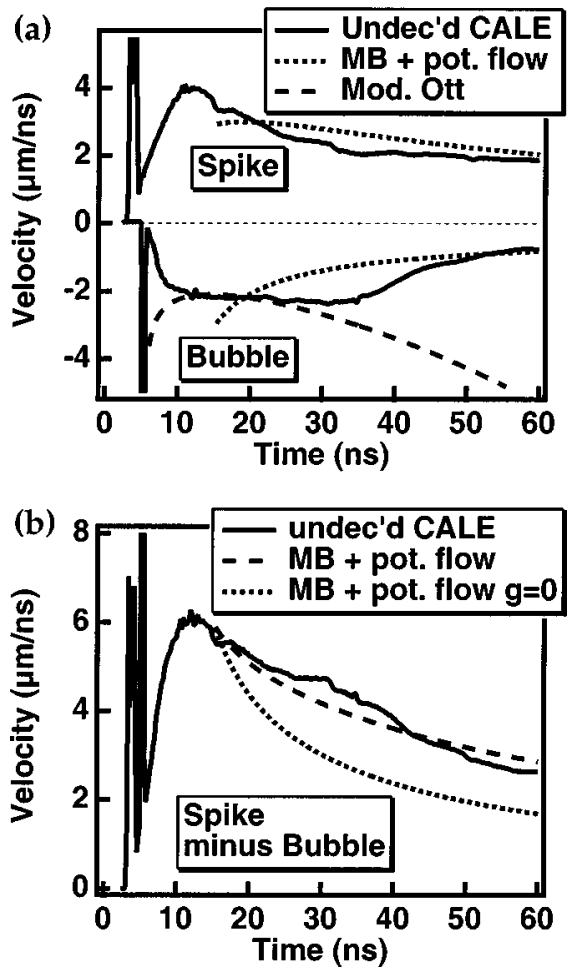

FIG. 5. Ott and potential flow theory.

bility due to the shock and the RT instability due to the deceleration, while in the latter case we ignore the deceleration and show the prediction for the pure RM case. The RM plus RT case predicts the velocity difference much better than the pure RM case; it appears that the instability growth is roughly 50\% RM-driven and 50\% RT-driven. In general, then, potential flow theory appears to describe the undecompressed peak-to-valley velocity, while a modification of the Ott thin-shell theory appears to describe the bubble velocity at early times. This result can be understood if at early times the thin-shell effect is strong, while at later times, the effect has weakened as the shell decompresses and widens.

\section{SCALING}

We can rigorously transform the hydrodynamics at the microscopic scale of a laser experiment to the astronomical scale of a $\mathrm{SN}$. We can do so because in both cases, the hydrodynamics is described by equations which scale from one regime to the other. Both the laser experiment and the $\mathrm{SN}$ at intermediate times (the first few hours) are described by compressible hydrodynamics equations - the Euler equations. This is true because in both cases the plasmas are collisional (particles are localized on small scales), viscosity is unimportant (inertial forces are much greater than viscous forces), thermal conduction is negligible (compared to advection of heat), and radiation transport effects are negligible (again, compared to advection of heat). For a more complete discussion of these criteria, see Ref. 33, and for additional discussion of scaling see Refs. 12, 28, 29, and 34. Briefly, let $h, v$, and $\tau$ be a typical scale length, fluid velocity, and time scale for either regime. The plasmas are collisional because 
the ratio $l_{c} / h$ is small, where $l_{c}$ is the collisional mean free path; for the SN, $l_{c} / h<2 \times 10^{-12}$, and for the laser, $l_{c} / h$ $<1 \times 10^{-6}$. Viscosity is unimportant because the Reynolds number $\mathrm{Re} \equiv h v / \nu$ is very large $\left(\gg 10^{3}\right)$, where $\nu$ is the dominant viscosity. For the $\mathrm{SN}$, photon viscosity dominates and $\operatorname{Re}>10^{13}$; for the laser experiment, ion viscosity dominates and $\operatorname{Re}>2 \times 10^{5}$. Thermal conduction is negligible because the Peclet number $\mathrm{Pe} \equiv h v / \chi \gg 1$, where $\chi$ is the thermal diffusivity. In both cases, magnetic fields are unimportant and $\chi$ is the thermal diffusivity for unmagnetized electrons. For the $\mathrm{SN}, \mathrm{Pe}>10^{11}$; for the laser experiment, $\mathrm{Pe}>2.5 \times 10^{3}$. That radiation transport is unimportant in both cases can be seen by considering either the photon Peclet number $\mathrm{Pe}_{\gamma}$ $\equiv h v / \chi_{\gamma}$, where $\chi_{\gamma}$ is the thermal diffusivity for photons, or the ratio $\tau_{R} / \tau$, where $\tau_{R}$ is the radiation cooling time. For the SN, $\mathrm{Pe}_{\gamma}>5 \times 10^{4}$; for the laser experiment, $\tau_{R} / \tau>500$.

The Euler equations (see Ref. 35),

$$
\begin{aligned}
& \frac{\partial}{\partial t} \rho+\nabla \cdot(\rho \mathbf{v})=0 \\
& \frac{\partial}{\partial t}(\rho \mathbf{v})+\nabla\left(P+\rho v^{2}\right)=0 \\
& \frac{\partial}{\partial t}(\rho E)+\nabla \cdot[(\rho E+P) \mathbf{v}]=0,
\end{aligned}
$$

where

$$
E=u+\frac{1}{2} v^{2},
$$

$u$ being the internal energy, are invariant under any scale transformation that preserves the quantity (space/ time $) \times(\text { density/pressure })^{1 / 2}$, i.e., $\rho=a \cdot \rho_{1}, \quad P=b \cdot P_{1}, \quad h$ $=c \cdot h_{1}, \tau=(a / b)^{1 / 2} c \tau_{1}$, where $h$ is a typical scale length, and $a, b$, and $c$ are constants. We assume here that in the regimes of interest, the equations of state are modeled reasonably well by polytropic gas laws with comparable adiabatic exponents (for further details, see Ref. 31). In the He-H layer of the $\mathrm{SN}$ at a time $\tau_{1}=1600 \mathrm{~s}$, we have $\rho_{1}=5$ $\times 10^{-3} \mathrm{~g} / \mathrm{cm}^{3}, P_{1}=100 \mathrm{Mbar}$, and $h_{1}=10^{12} \mathrm{~cm}$. In the compressible laser experiments, we have $\rho=4 \mathrm{~g} / \mathrm{cm}^{3}, \quad P$ $=1 \mathrm{Mbar}$, and $h=200 \mu \mathrm{m}$. We use the transformation to find the remaining undetermined quantity, $\tau$, the characteristic time scale for the compressible laser experiment. Determining the constants of the transformation, we find that $a$ $=\rho / \rho_{1}=8 \times 10^{2}, \quad b=P / P_{1}=10^{-2}, \quad$ and $\quad c=h / h_{1}=2$ $\times 10^{-14}$. Thus the density, pressure, and spatial scales differ by 2, 2, and 14 orders of magnitude, respectively. Applying the transformation, we find that a typical scale time $\tau$ is 20 $\mathrm{ms}$, by which time the degree of instability growth in the laser experiment is indeed similar to what we see in a simulation of the He-H layer of the $\mathrm{SN}$ on a time scale of $\approx 10^{3} \mathrm{~s}$. Thus for the case of compressible hydrodynamics described by the Euler equation, we can scale the hydrodynamics from the laser experiment to the SN.

\section{2D VS 3D HYDRO}

We are beginning experimental investigations of 2D vs 3D SN-relevant hydrodynamics in experiments on the Nova
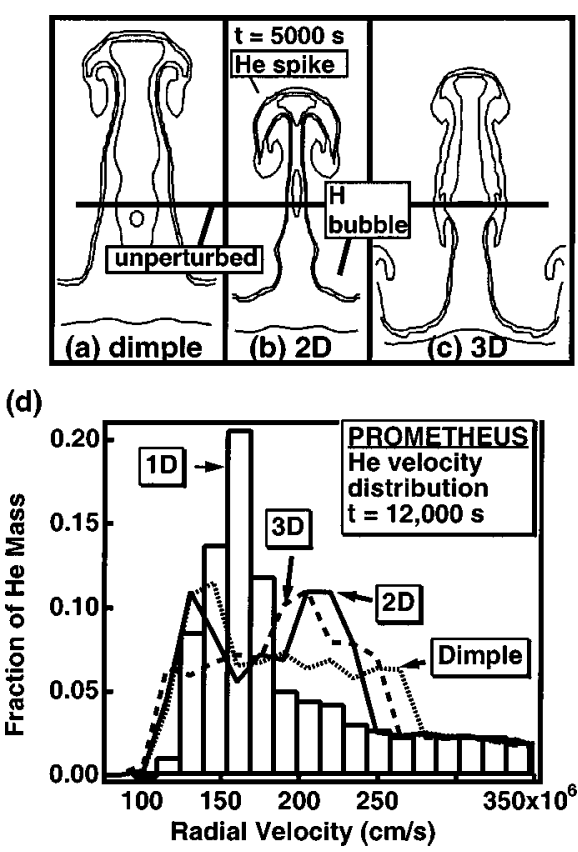

FIG. 6. (a), (b), and (c) He-H interface growth for dimple, 2D, and 3D perturbations; (d) He velocity distributions.

and Omega lasers. 2D vs 3D differences could help explain why 2D simulations of SN 1987A underpredict the observed ${ }^{56}$ Co velocities. 3D numerical simulations are very expensive to carry out routinely, and intractable at the higher resolutions used in 2D simulations such as the ones presented in Figs. 3(c)-3(d). However, a laser experiment with a 3D perturbation is no more difficult to perform than an experiment with a 2D perturbation, and thus offers a useful tool for studying SN-relevant hydro. In the linear regime, the growth rates of a 3D perturbation and a $2 \mathrm{D}$ perturbation are the same when both perturbations have the same wave number, and the $3 \mathrm{D}$ perturbation grows faster in the nonlinear regime than the $2 \mathrm{D}$ perturbation, because the growth saturates later in $3 \mathrm{D}$ (see Ref. 36 and references therein). The later saturation in 3D is explained by drag-vs-buoyancy arguments (see Refs. 28,29 , and 37). In both the linear and nonlinear regimes, the term "growth" means the time rate of change of the amplitude of the perturbation, either from unperturbed interface to bubble or spike tip, or from bubble to spike tip. Growth in the linear regime is typically described by a growth rate (see Ref. 36, for example), and we quantify growth in the nonlinear regime by velocities of the bubble-and-spike tips.

We are currently investigating both "dimple" $\left(J_{0}\right.$ Bessel) and 3D $[\sin (k x) \times \sin (k y)]$ interface perturbations [as opposed to the 2D sinusoidal corrugation in Fig. 3(a)]. We are also doing numerical simulations to investigate 2D-3D differences in SN 1987A. Figure 6 illustrates a simple investigation, comparing the result of imposing 2D sinusoidal, dimple, and 3D velocity perturbations at the He-H interface after passage of the shock through the interface. The 3D bubble [Fig. 6(c)] grows faster than the 2D bubble [Fig. 6(b)], a result already anticipated from theoretical work (see Ref. 36 and references therein) and from previous Nova experiments (see Ref. 37 ), and the spike grows $\approx 25 \%$ faster in 


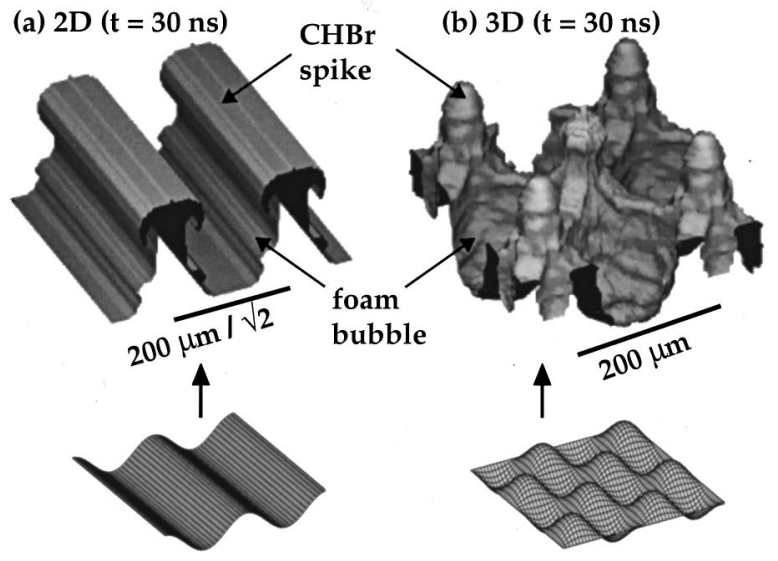

(c)

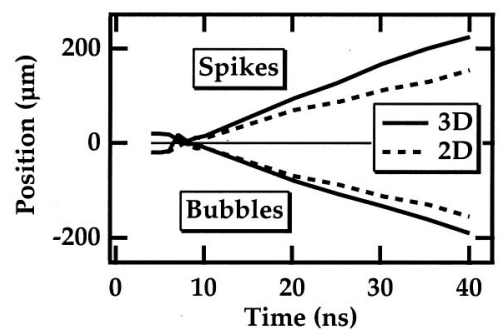

FIG. 7. Indirect drive Nova SNRT 2D sine versus 3D crosshatch. (a) 2D sinusoid; initial perturbation and interface at $40 \mathrm{~ns}$; (b) same for crosshatch; (c) bubble-and-spike positions versus time. Note the greater penetration of the $3 \mathrm{D}$ spike.

3D. The dimple spike [Fig. 6(a)] grows significantly faster than the 2D spike, while the dimple bubble ring, which had a lower initial amplitude than the 2D bubble, grows more slowly than the 2D bubble. Figure 6(d) shows the distribution by mass of the He velocities at $12000 \mathrm{~s}$, by which time the blast wave has exited the star. The broadening of the velocity profiles in the He layer for the $3 \mathrm{D}$ and dimple perturbations is evident. The $\mathrm{H}$ layer is $\approx 20 \% \mathrm{He}$ by mass in the initial model, so the $\mathrm{He}$ at the higher velocities is in the outer layer of the star and has a similar velocity distribution in all cases.

In the Nova experiments, illustrated in Fig. 7, we are comparing 3D interface perturbations of the type $\sin (k x)$ $\times \sin (k y)$ to the $2 \mathrm{D}$ sinusoidal corrugation. The target consists of a $200 \mu \mathrm{m}$ thick $\mathrm{CHBr}$ ablator of density $1.54 \mathrm{~g} / \mathrm{cm}^{3}$, backed by a thick layer of foam of density $100 \mathrm{mg} / \mathrm{cm}^{3}$. We again use Nova to produce an X-ray drive that shocks the target. We use PROMETHEUS to model the experiment, because PROMETHEUS allows three-dimensional geometries (see the description of the codes in Sec. I). We use only low resolution, 24 zones per half wavelength, because of the expense of the 3D simulations. For the 2D sinusoid, we use $\lambda=200 \mu \mathrm{m} / 2^{1 / 2}$ and for the 3D "crosshatch" we use $\lambda$ $=200 \mu \mathrm{m}$, which gives the same wave number $k$ for both cases. From the PROMETHEUS simulation, we find that the 3D spike grows about $35 \%$ faster than the the 2D spike, which should be experimentally observable.

We will compare a Bessel dimple perturbation to the 2D sinusoid in the experiments at the Omega laser. In the Omega laser shots we will directly illuminate the target with
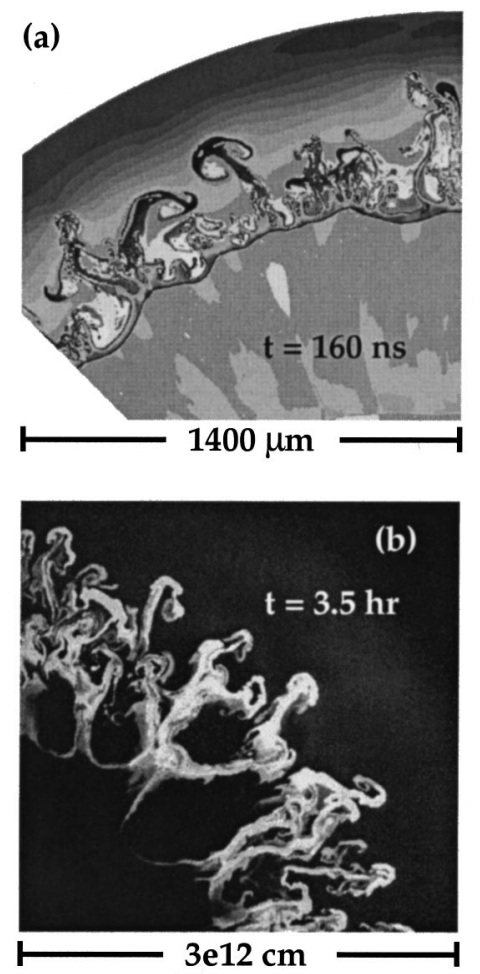

FIG. 8. Further work (a) CALE simulation of three-layer spherical geometry laser experiment designed for the National Ignition Facility; (b) PROMETHEUS simulation of hydrodynamic instabilities in SN 1987A; image reproduced from Ref. 22.

the laser, instead of using the laser to generate an $\mathrm{x}$-ray drive. The target will consist of $50 \mu \mathrm{m}$ of $\mathrm{CH}_{2}$ backed by $150 \mu \mathrm{m}$ of $\mathrm{CHBr}$, with a lighter layer of foam. The $\mathrm{CH}_{2}$ layer facing the laser prevents preheating of subsequent layers by transmitted x-rays. We use CALE to simulate this experiment, since CALE has both planar and axially symmetric $r-z$ geometry (see the discussion of the codes in Sec. I). In the CALE simulations (not shown) the Bessel spike grows approximately $35 \%$ faster than the $2 \mathrm{D}$ spike, whereas the Bessel bubble ring, having a lower initial amplitude than the 2D bubble, grows to lower amplitude than the $2 \mathrm{D}$ bubble.

\section{FURTHER WORK}

We are also starting to design targets which incorporate more features of the actual star, in particular divergent geometry and multiple layers of different density. Figure 8(a) shows a CALE simulation of a spherical geometry three-layer target with multimode perturbations at the initial interfaces. Material from the lowest layer has been mixed well into the outer layer by the interacting instabilities at the two interfaces. Such mixing is reminiscent of the mixing of the $O$ layer into the $H$ envelope in SN 1987A, illustrated in Fig. 8(b) by a PROMETHEUS simulation of SN 1987A. This target is being designed for SN experiments on the National Ignition Facility, now under construction. 


\section{ACKNOWLEDGMENTS}

Work performed under the auspices of the U.S. Department of Energy by the Lawrence Livermore National Laboratory under Contract No. W-7405-ENG-48. D.A. and J.K. were supported in part by NASA Grant No. NAGW-2450 and NSF Grant No. ASTRO 9015976. We are grateful to D. Shvarts and D. Oron for ready assistance in our potential flow modeling.

${ }^{1}$ R. D. Richtmyer, Commun. Pure Appl. Math. 13, 297 (1960).

${ }^{2}$ E. E. Meshkov, Izv. Akad. Nauk SSSR, Mekh. Zhidk. Gaza 4, 151 (1969); Izv., Acad. Sci., USSR Fluid Dyn. 4, 101 (1969).

${ }^{3}$ Lord Rayleigh, Scientific Papers II, Cambridge, England, 1900.

${ }^{4}$ G. Taylor, Proc. R. Soc. London, Ser. A 201, 192 (1950).

${ }^{5}$ D. Arnett, J. N. Bahcall, R. A. Kirshner, and S. E. Woosley, Annu. Rev. Astron. Astrophys. 27, 629 (1989).

${ }^{6}$ T. Shigeyama and K. Nomoto, Astrophys. J. 360, 242 (1990).

${ }^{7}$ R. W. Hanuschik and J. Dachs, Astron. Astrophys. 192, L29 (1987).

${ }^{8}$ V. M. Blanco et al., Astrophys. J. 320, 589 (1987).

${ }^{9}$ F. C. Witteborn, J. D. Bregman, D. H. Wooden, P. A. Pinto, D. M. Rank, S. E. Woosley, and M. Cohen, Astrophys. J. 338, L9 (1989).

${ }^{10}$ J. Tueller, S. Barthelmy, N. Gehrels, B. J. Teegarden, M. Leventhal, and C. J. MacCallum, Astrophys. J. 351, L41 (1990).

${ }^{11}$ R. McCray, Annu. Rev. Astron. Astrophys. 31, 175 (1993).

${ }^{12}$ J. Kane, D. Arnett, B. A. Remington, S. G. Glendinning, J. Castor, R. Wallace, A. Rubenchik, and B. A. Fryxell, Astrophys. J. 478, L75 (1997).

${ }^{13}$ B. A. Remington et al., Phys. Plasmas 4, 1994 (1997).

${ }^{14}$ R. P. Drake et al., Phys. Rev. Lett. 81, 2068 (1998).

${ }^{15}$ R. P. Drake, J. P. Carroll, K. Estabrook, S. G. Glendinning, B. A. Remington, R. Wallace, and R. McCray, Astrophys. J., 500, L157 (1998).

${ }^{16}$ J. T. Larsen and S. M. Lane, J. Quant. Spectrosc. Radiat. Transf. 51, 179 (1994).

${ }^{17}$ R. T. Barton, in Numerical Astrophysics, edited by J. M. Centrella, J. M.
LeBlanc, and R. L. Bowers (Jones and Bartlett, Boston, 1985), pp. 482497.

${ }^{18}$ P. R. Woodward and P. Colella, J. Comput. Phys. 54, 115 (1984).

${ }^{19}$ D. Arnett, Supernovae and Nucleosynthesis (Princeton University Press, Princeton, 1996).

${ }^{20}$ D. Arnett, B. A. Fryxell, and E. Müller, Astrophys. J. 341, L63 (1989).

${ }^{21}$ B. A. Fryxell, E. Müller, and D. Arnett, Astrophys. J. 367, 619 (1991).

${ }^{22}$ E. Müller, B. A. Fryxell, and D. Arnett, Astron. Astrophys. 251, 505 (1991).

${ }^{23}$ S. G. Glendinning, Rev. Sci. Instrum. 63, 5108 (1992)

${ }^{24}$ G. M. Dimonte, C. E. Frerking, M. Schneider, and B. A. Remington, Phys. Plasmas 3, 614 (1996).

${ }^{25}$ K. S. Budil, B. A. Remington, T. A. Peyser, K. O. Mikaelian, P. L. Miller, N. C. Woolsey, W. M. Wood-Vasey, and A. M. Rubenchik, Phys. Rev. Lett. 76, 4536 (1996).

${ }^{26}$ T. A. Peyser, P. L. Miller, P. E. Stry, K. S. Budil, E. W. Burke, D. A. Wojtowicz, D. L. Griswold, B. A. Hammel, and D. W. Phillion, Phys. Rev. Lett. 75, 2332 (1995).

${ }^{27}$ U. Alon, J. Hecht, D. Mukamel, and D. Shvarts, Phys. Rev. Lett. 72, 2867 (1994).

${ }^{28}$ U. Alon, J. Hecht, D. Ofer, and D. Shvarts, Phys. Rev. Lett. 74, 534 (1995).

${ }^{29}$ J. Hecht, U. Alon, and D. Shvarts, Phys. Fluids 6, 4019 (1994).

${ }^{30}$ E. Ott, Phys. Rev. Lett. 29, 1429 (1995).

${ }^{31}$ M. M. Basko, Phys. Plasmas 1, 1270 (1994).

${ }^{32}$ K. A. Mayer and P. J. Blewett, Phys. Fluids 15, 753 (1972).

${ }^{33}$ D. Ryutov, R. P. Drake, J. Kane, E. Liang, B. A. Remington, and W. M. Wood-Vasey, "Similarity criteria for the laboratory simulation of supernova hydrodynamics," Astrophys. J., June 1999 (in press).

${ }^{34}$ E. M. Campbell et al., Laser Part. Beams 15, 607 (1997).

${ }^{35}$ L. Landau and E. Lifshitz, Fluid Dynamics (Pergammon, Princeton, 1960).

${ }^{36}$ J. P. Dahlburg, J. H. Gardner, G. D. Doolen, and S. W. Haan, Phys. Fluids B 5, 571 (1993).

${ }^{37}$ M. M. Marinak et al., Phys. Rev. Lett. 75, 3677 (1995). 